\title{
Aortic valve-mediated wall shear stress is heterogeneous and predicts regional aortic elastic fiber thinning in bicuspid aortic valve-associated aortopathy
}

Emilie Bollache, PhD, ${ }^{\mathrm{a}}$ David G. Guzzardi, PhD, ${ }^{\mathrm{b}}$ Samaneh Sattari, MSc, ${ }^{\mathrm{c}}$ Katherine E. Olsen, BEng, Elena S. Di Martino, PhD, ${ }^{\mathrm{d}}$ S. Chris Malaisrie, MD, ${ }^{\mathrm{e}}$ Pim van Ooij, PhD, ${ }^{\mathrm{f}}$ Jeremy Collins, MD, ${ }^{\mathrm{a}}$ James Carr, MD, ${ }^{\mathrm{a}}$ Patrick M. McCarthy, MD, ${ }^{\mathrm{e}}$ Michael Markl, PhD, ${ }^{\mathrm{a}, \mathrm{g}}$ Alex J. Barker, PhD, ${ }^{\mathrm{a}}$ and Paul W. M. Fedak, MD, PhD, FRCSC ${ }^{\text {,e }}$

\section{ABSTRACT}

Objectives: The objectives of this study were to investigate an association between the magnitude of flow-mediated aortic wall shear stress (WSS) and medial wall histopathology in patients with bicuspid aortic valve (BAV) with aortopathy.

Methods: Patients with BAV ( $\mathrm{n}=27 ; 52 \pm 15$ years; 3 women; proximal thoracic aorta diameter $=4.4 \pm 0.7$ and $4.6 \pm 0.5 \mathrm{~cm}$ ) who underwent prophylactic aortic resection received preoperative 3 -dimensional time-resolved phase-contrast magnetic resonance imaging with 3-dimensional velocity encoding to quantify WSS relative to a population of healthy age- and sex-matched tricuspid aortic valve control participants $(n=20)$. Quantitative histopathology was conducted on BAV aorta tissue samples resected at surgery $(n=93)$, and correlation was performed between elastic fiber thickness and in vivo aortic WSS as continuous variables. Validation of elastic fiber thickness was achieved by correlation relative to tissue stiffness determined using biaxial biomechanical testing ( $\mathrm{n}=22$ samples).

Results: Elastic fibers were thinner and WSS was higher along the greater curvature compared with other circumferential regions (vs anterior wall: $P=.003$ and $P=.0001$, respectively; lesser curvature: both $P=.001$ ). Increased regional WSS was associated with decreased elastic fiber thickness $(r=-0.25 ; P=.02)$. Patient stratification with subanalysis showed an increase in the correlation between WSS and histopathology with aortic valve stenosis $(r=-0.36 ; P=.002)$ and smaller aortic diameters $(<4.5 \mathrm{~cm}: r=-0.39 ; P=.03)$. Elastic fiber thinning was associated with circumferential stiffness $(r=-0.41 ; P=.06)$.

Conclusions: For patients with BAV, increased aortic valve-mediated WSS is significantly associated with elastic fiber thinning, particularly with aortic valve stenosis and in earlier stages of aortopathy. Elastic fiber thinning correlates with impaired tissue biomechanics. These novel findings further implicate valve-mediated hemodynamics in the progression of BAV aortopathy. (J Thorac Cardiovasc Surg 2018;156:2112-20)

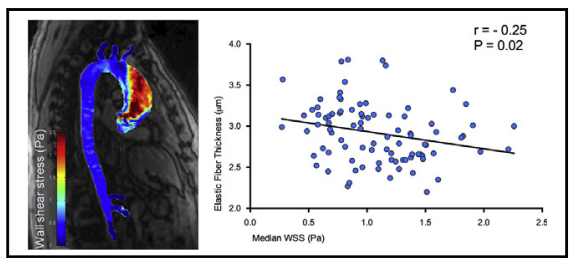

In vivo wall shear stress correlates with local elastic fiber thinning in the BAV aorta.

\section{Central Message}

Increased aortic wall shear stress is associated with elastic fiber thinning in bicuspid aortopathy, and is most pronounced with aortic valve stenosis and mildly dilated aortas.

\section{Perspective}

For the first time in bicuspid aortopathy, we report direct correlation between quantitative in vivo aortic wall shear stress and elastic fiber histopathology. Histopathology corresponds with impaired tissue biomechanics, and implicates abnormal bicuspid aortic valve-mediated hemodynamics in aortopathy progression. Research into flow imaging for noninvasive biomarker risk stratification is warranted.

See Editorial Commentaries pages 2121 and 2123.

See Editorial page 2110.
From the ${ }^{\mathrm{a}}$ Department of Radiology, Feinberg School of Medicine, ${ }^{\mathrm{g}}$ Department of Biomedical Engineering, McCormick School of Engineering, Northwestern University, Chicago, Ill, ${ }^{\mathrm{b}}$ Department of Cardiac Sciences, Cumming School of Medicine, ${ }^{\mathrm{d}}$ Department of Civil Engineering, Schulich School of Engineering, Libin Cardiovascular Institute, ' Graduate Program in Biomedical Engineering, University of Calgary, Calgary, Alberta, Canada, ${ }^{\mathrm{e} D i v i s i o n}$ of Surgery-Cardiac Surgery, Northwestern University, Bluhm Cardiovascular Institute, Chicago, Ill, and ${ }^{\mathrm{f}} \mathrm{De}-$ partment of Radiology, Academic Medical Center, Amsterdam, The Netherlands. This work was supported by the National Institutes of Health grants R01HL115828 (M.M.), R01HL133504 (A.J.B., P.W.M.F.) and K25HL119608 (A.J.B.), the American Heart Association Midwest Affiliate grant 16POST27250158 (E.B.), an Alberta Innovates Health Solutions MD-PhD Studentship (D.G.G.), and by the
Melman Bicuspid Aortic Valve Program, Bluhm Cardiovascular Institute (P.W.M.F.). Funding sources played no part in data analysis or interpretation. Drs Bollache and Guzzardi share co-first authorship.

Received for publication Feb 17, 2018; revisions received May 3, 2018; accepted for publication May 26, 2018; available ahead of print July 27, 2018.

Address for reprints: Paul W.M. Fedak, MD, PhD, FRCSC, Section of Cardiac Surgery, Department of Cardiac Sciences, Cumming School of Medicine, University of Calgary, Room 880, 1403-29 St NW, Calgary, Alberta T2N 2T9, Canada (E-mail: paul.fedak@gmail.com). $0022-5223 / \$ 36.00$

Copyright (C) 2018 by The American Association for Thoracic Surgery https://doi.org/10.1016/j.jtcvs.2018.05.095 


\section{Abbreviations and Acronyms \\ A $=$ anterior wall \\ $\mathrm{AA}_{\text {dist }} \quad=$ distal ascending aorta \\ $\mathrm{AA}_{\text {prox }}=$ proximal ascending aorta \\ AR $\quad=$ aortic regurgitation \\ AS $=$ aortic valve stenosis \\ BAV = bicuspid aortic valve}

4D flow MRI = three-dimensional time-resolved phase-contrast magnetic resonance imaging with 3-D velocity encoding

ECG = electrocardiogram

$\mathrm{GC}=$ greater curvature

$\mathrm{LC} \quad=$ lesser curvature

MRI $=$ magnetic resonance imaging

$\mathrm{P}=$ posterior wall

TAV $=$ tricuspid aortic valve

WSS $\quad=$ wall shear stress
Bicuspid aortic valve (BAV) is a common congenital cardiac malformation, affecting approximately $1 \%$ to $2 \%$ of the population. ${ }^{1}$ One-third of patients with BAV might develop serious complications leading to increased morbidity including aortic aneurysms with dissection or rupture. ${ }^{1}$ More than half of patients with BAV will require a medical or surgical intervention within 20 years of diagnosis. ${ }^{2}$ The mechanisms underlying BAV aortopathy remain unclear, and its probability of development and progression for individual patients is difficult to predict. There remains controversy regarding timing, and type and extent of surgery that should be performed for bicuspid aortopathy and poor compliance with practice guidelines. ${ }^{3}$ Expert consensus has also been variable; 10 different surgical guidelines were published between 1998 and 2014. ${ }^{4}$ Together with the doubling of BAV surgical interventions over the past decade at an estimated cost of over 1 billion USD, ${ }^{5}$ an improved understanding of BAV aortopathy is needed.

Despite widespread assumption that BAV-related aortic wall remodeling is associated with an underlying genetic connective tissue defect, increasing evidence also implicates abnormal valve-mediated hemodynamics of the ascending aorta in BAV aortopathy progression. ${ }^{6}$ Phase-contrast magnetic resonance imaging (MRI) with 3-D velocity encoding and resolution over time (cine) along the cardiac cycle (known as "4D flow MRI"), has emerged as a powerful noninvasive imaging technique to investigate complex 3-D proximal aorta blood flow patterns. ${ }^{7}$ Altered valve-mediated flow can increase wall shear stress (WSS), which represents the tangential viscous force exerted by blood flow on the arterial wall. WSS is a known trigger of outward vascular remodeling via endothelial mechanotransduction and might become a useful hemodynamic biomarker of BAV aortopathy risk and severity. ${ }^{8}$ Noninvasive $4 \mathrm{D}$ flow MRI showed increased valve-mediated WSS in patients with normally functioning BAV compared with patients with a tricuspid aortic valve (TAV). ${ }^{9}$ This indicates that all patients with BAV have altered proximal aortic valve-mediated hemodynamics, which might mediate the associated aortopathy.

We recently documented more severe medial elastic fiber degradation in resected aortic tissue from regions of increased WSS compared with adjacent regions of normal WSS in the same aortae of 20 patients with BAV. ${ }^{10}$ This observation supports 4D flow MRI of valve-mediated hemodynamics as a noninvasive method to identify aortic tissue at risk for aortopathy progression. However, our past work was limited by analyzing only 2 adjacent aortic tissue regions (although many were collected) for each patient and WSS was analyzed as a categorical variable (increased WSS: "hot" vs normal: "cold" regions). We present a more comprehensive study on the relationship in BAV between valve-mediated hemodynamics and aortic wall histopathology using WSS as a continuous variable and by investigating the influence of key patient-specific factors on this relationship. Metrics of histopathology were further validated by assessing biomechanical tissue stiffness.

\section{METHODS}

\section{Study Population}

Informed consent was obtained for 27 patients with BAV who received ascending aortic surgery between January 2012 and July 2015. Patients were prospectively recruited to undergo preoperative MRI and aortic tissue resection during surgery with institutional review board approval (REB17-0207; October 6, 2016). Preoperative continuous-wave Doppler ultrasound was used to classify severity of aortic valve stenosis (AS) according to systolic peak velocity (mild: $2-3 \mathrm{~m} / \mathrm{s}$; moderate-severe: $\geq 3 \mathrm{~m} / \mathrm{s}$ ), and aortic regurgitation (AR) according to regurgitant fraction (mild: $<30 \%$; moderate-severe: $\geq 30 \%$ ). ${ }^{11}$ Additionally, a control group of 20 healthy (nonsurgical) volunteers with normal TAV and aorta was randomly identified to match the patient group for age and sex from a local 4D flow MRI database of 1673 subjects, including 105 healthy volunteers, via institutional review board-approved retrospective chart review with a consent waiver.

\section{Aortic Tissue Collection}

Aortic tissue samples $(n=93)$ were collected during surgery as before, ${ }^{10}$ and classified according to their longitudinal and circumferential location. The ascending aorta between the level of the inferior border of the right pulmonary artery and the proximal takeoff of the brachiocephalic artery was equally divided in 2 , to define the proximal $\left(\mathrm{AA}_{\text {prox }}\right)$ and distal $\left(\mathrm{AA}_{\text {dist }}\right)$ ascending aortic longitudinal zones (Figure 1, A). Each zone was 
subdivided into 4 circumferential regions: the anterior $(\mathrm{A})$ and posterior $(\mathrm{P})$ walls, as well as the greater (GC) and lesser (LC) curvatures (Figure 1, B).

\section{Histopathology: Elastic Fiber Architecture}

Circumferential Verhoeff-Van Gieson histological staining of each tissue sample and analysis of elastic fiber architecture was performed as previously described. ${ }^{10,12}$ All analyses were conducted using fiber elastic thickness as the continuous variable.

\section{Tensile Mechanical Testing: Tissue Stiffness}

To validate elastic fiber thickness as an aortopathy metric, all samples of resected bicuspid aorta tissue of sufficient size (approximately $1 \mathrm{~cm}^{2} ; \mathrm{n}=22$, collected as described previously) from each aortic region of the 27 patients with BAV were subjected to biaxial mechanical testing. ${ }^{13}$ Results were correlated to elastic fiber thickness. Because of its putative role in aortopathy pathogenesis, ${ }^{14}$ circumferential maximum tangential stiffness of the samples were computed. Testing details, analysis, and modeling of the experimental data are provided in Appendix E1.

\section{MRI Acquisitions: Aortic Wall Shear Stress Quantification}

All patient and control MRI exams were performed between 2011 and 2016. Standard of care thoracic exam acquisition in patients included electrocardiogram (ECG)-gated 2-dimensional cine balanced steady-state free precession images covering the left ventricle for stroke volume and ejection fraction evaluation, and at the aortic valve for Sievers BAV morphology classification. ${ }^{15}$ Additionally, contrast-enhanced magnetic resonance angiography of the thoracic aorta was used to calculate aortic diameters at the sinuses of Valsalva, sinotubular junction, mid-ascending aorta, as well as proximal and distal arch to classify patients according to the Stanford-Fazel BAV aortopathy clusters. ${ }^{16}$ Finally, a prospective ECG- and respiratory navigator-gated aortic 4D flow MRI was performed in all patients and controls to derive WSS. Details regarding MRI acquisition parameters and data analysis are provided in Appendix E1.

Briefly, after conventional preprocessing of each 4D flow MRI data set and segmentation of the 3-D aortic volume (Appendix E1), peak systole was automatically detected as the frame with the highest velocity averaged over the whole volume. Peak systolic WSS vectors were then calculated throughout the entire 3-D aortic surface as blood dynamic viscosity multiplied by the velocity spatial gradient at the wall. ${ }^{17}$ Local median WSS magnitude was extracted in regions of interest matched to the wall location of resected BAV tissue samples. An in-house Matlab (MathWorks, Natick, Mass) interface provided aortic WSS map visualization in the 3 main orientations (sagittal, coronal, axial) superimposed to magnitude anatomical images, drawing of regions of interest in any view along with real-time adjustment in the 2 other orthogonal views, to define the $\mathrm{AA}_{\text {prox }}$ and $\mathrm{AA}_{\text {dist }}$ longitudinal zones and A, P, GC, and LC circumferential regions (Figure 1).

\section{Statistical Analysis}

Lilliefors test was used to assess whether data were normally distributed. Data are reported as mean \pm SD when normally distributed, or as median (interquartile range) otherwise. The Wilcoxon rank sum test was used to assess differences in elastic fiber thickness, WSS between aortic regions, and differences between BAV patients and healthy volunteers, as well as stratification between patient groups according to AS and aortic diameters. Associations between elastic fiber thickness, WSS, and tissue stiffness were correlated, and Pearson correlation coefficients $r$ are reported. Statistical analyses were performed using Matlab (MathWorks); $P<.05$ was considered statistically significant.

\section{RESULTS \\ Study Cohort}

Patient demographic and preoperative characteristics are summarized in Table 1. Twenty-six of the 27 patients
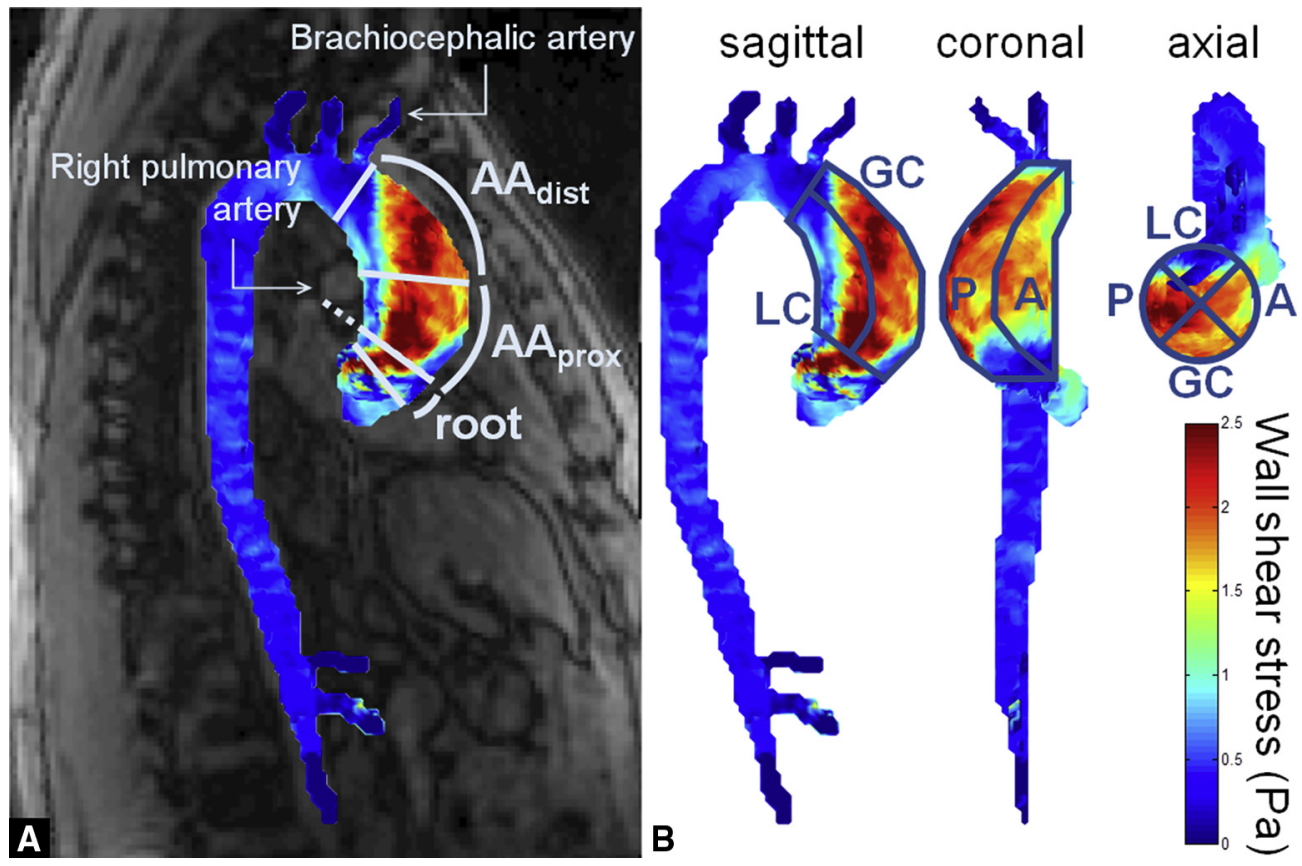

FIGURE 1. Example of 3-dimensional time-resolved phase-contrast magnetic resonance imaging with 3-dimensional velocity encoding-derived aortic wall shear stress map in a patient with bicuspid aortic valve and definition of aortic regions of interest matching resected tissue sample location. A, Two longitudinal zones were defined as the proximal $\left(A A_{\text {prox }}\right)$ and distal $\left(A A_{\text {dist }}\right)$ ascending aorta, using anatomical landmarks from magnitude images $($ details in text). B, Circumferential regions ( $G C$, Greater curvature; $L C$, lesser curvature; $P$, posterior; and $A$, anterior) were defined using the sagittal, coronal, and axial views. 
TABLE 1. Preoperative characteristics of patients with BAV $(n=27)$

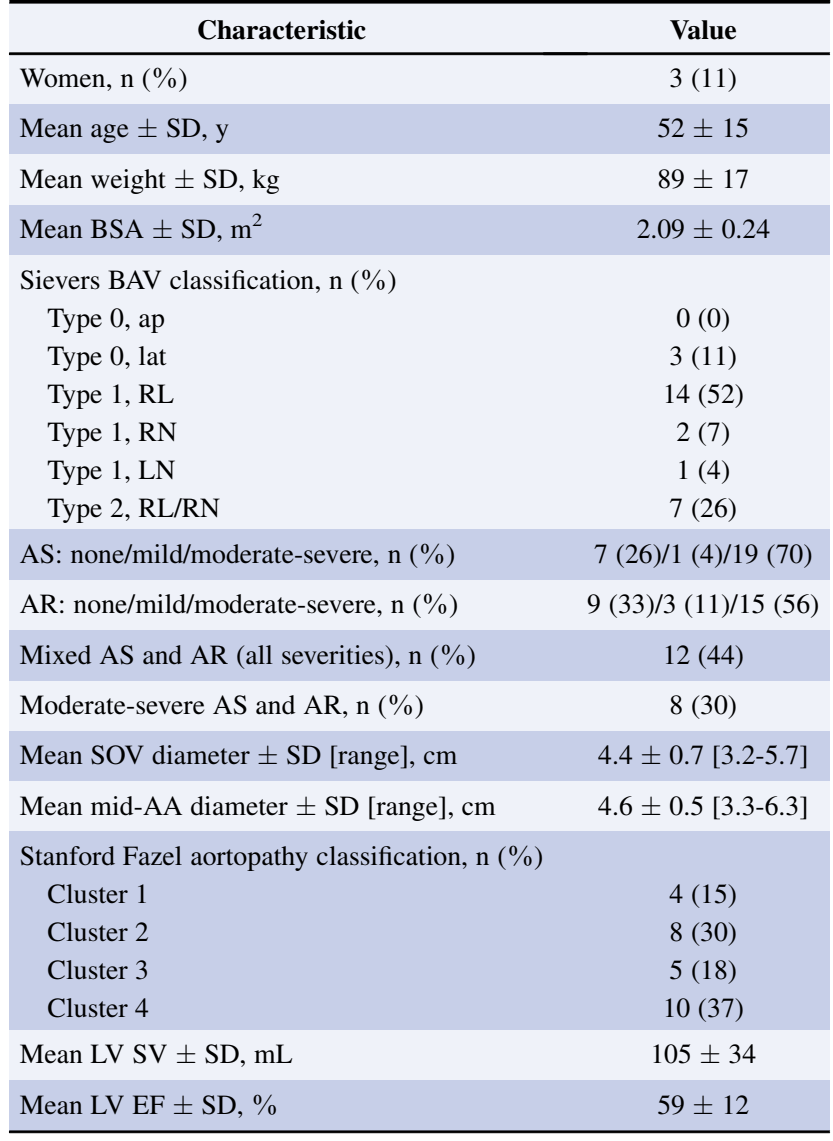

$S D$, Standard deviation; $B S A$, body surface area; $B A V$, bicuspid aortic valve; $a p$, anterior-posterior; lat, lateral; $R L$, right-left coronary sinus; $R N$, right-non coronary sinus; $L N$, left-non coronary sinus; $A S$, aortic stenosis; $A R$, aortic regurgitation; $S O V$, sinus of Valsalva; $A A$, ascending aorta; $L V$, left ventricular; $S V$, stroke volume; $E F$, ejection fraction.

with BAV (96\%) had either AS and/or AR: although 1 patient had neither, the remaining 6 patients without significant AS had moderate-severe AR (predominant AR), and the remaining 8 patients without significant $A R$ had moderate-severe AS (predominant AS). Healthy ageand sex-matched controls $(\mathrm{n}=20 ; 2$ women; age: $48 \pm 14$ years; weight: $95 \pm 19 \mathrm{~kg}$; height: $175 \pm 5 \mathrm{~cm}$ ) provided a physiologic normal range of WSS values.

\section{Histopathology}

A median number of 3 (range, 1-11) aortic tissue samples were collected in patients with BAV from the tubular ascending aorta (as limited by the extent of surgical resection), yielding a total of $n=93$ samples (Table E1). Most samples $(\mathrm{n}=81)$ were collected within the $\mathrm{AA}_{\text {prox }}$, which was resected in all but 1 patient. Because of the lower number of samples collected in the $\mathrm{AA}_{\text {dist }}(\mathrm{n}=12)$, further results are presented over the entire ascending aorta (ie, $\left.\mathrm{AA}_{\text {prox }}+\mathrm{AA}_{\text {dist }}\right)$. Elastic fiber thickness could not be assessed with histology in $\mathrm{n}=3$ samples (longitudinal zone-circumferential region: $\mathrm{AA}_{\text {prox }}-\mathrm{A}, \mathrm{n}=1 ; \mathrm{AA}_{\text {prox }}-\mathrm{P}$, $\mathrm{n}=2$ ) because of the small size of these samples. Figure 2 provides indices of elastic fiber thickness averaged over the 27 patients with BAV in the remaining samples, according to circumferential region. Medial elastic fibers were significantly thinner in the GC compared with the A $(P=.003)$ and LC $(P=.001$; Table 2$)$. Stratification according to valve dysfunction or aortic size showed no differences in severity of elastic fiber histopathology (Table 2).

\section{D Flow MRI-Derived Regional Aortic WSS}

4D flow MRI was successfully acquired in all 27 preoperative patients with BAV and 20 healthy volunteers. Aortic WSS was calculated in all regions where BAV aortic tissue samples were collected: across all 4 circumferential regions in all $\mathrm{AA}_{\text {prox }}$ and $\mathrm{AA}_{\text {dist }}$ longitudinal zones, as well as in all corresponding regions for each control. Median aortic WSS on the GC of healthy volunteers was significantly lower than on the $\mathrm{LC}(P=.04$; Table 2 and Figure 3$)$. It was significantly increased in patients with BAV compared with the LC and A on the GC $(P=.001$ and $P=.0001$, respectively; Table 2 and Figure 3$)$ and $\mathrm{P}(P=.003$ and $P=.0006$, respectively; Table 2 and Figure 3). In a comparison of the 2 groups, patients with BAV had significantly higher aortic WSS than healthy volunteers at all locations except the LC (A: $P=.002$; P: $P=.0001$; GC: $P=.0001$; Table 2 and Figure 3). Stratification of patients with BAV for median aortic WSS according to AS or diameter revealed no significant differences between subgroups (Table 2).

\section{Relationship Between Histopathology and WSS}

A significant inverse relationship was observed between elastic fiber thickness and WSS $(r=-0.25 ; P=.02$; Figure 4, $A$, over all locations); increased WSS was associated with decreased elastic fiber thickness. The effects of AS and aortic diameter on the relationship between elastic

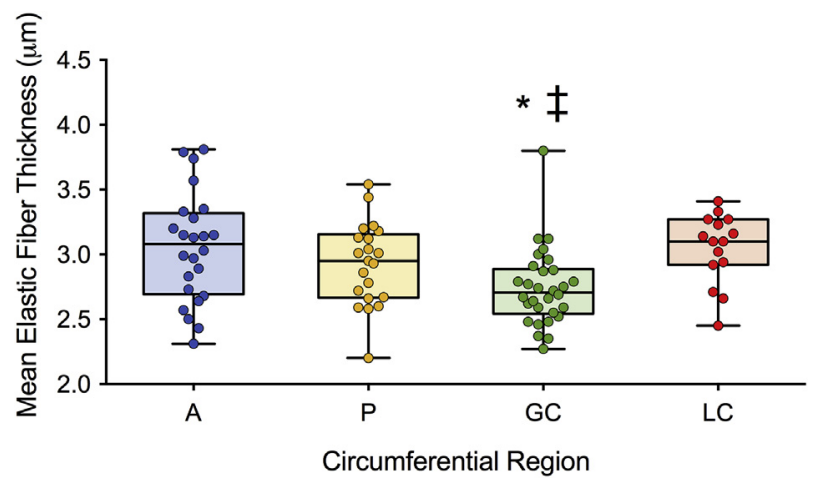

FIGURE 2. Aortic elastic fiber thickness assessed using histology in patients with bicuspid aortic valve (BAV) according to the circumferential location, while pooling the proximal and distal ascending aorta $(A$, Anterior; $P$, posterior, $G C$, greater curvature; and $L C$, lesser curvature). Columns illustrate median values whereas error bars indicate interquartile ranges. ${ }^{*} P<.05$ for comparison versus $\mathrm{A} A$; †versus LC. 
fiber thickness and median WSS in BAV patients are presented in Figure 4, $B$ and $C$. The association was stronger in subgroups of patients with predominant AS $(r=-0.36 ; P=.002$; Figure $4, B)$ and smaller aortic diameter $(<4.5 \mathrm{~cm}: r=-0.39 ; P=.03$; Figure $4, C)$ compared with correlation including all patients with BAV in the study. No significant associations between elastic fiber thickness and WSS were obtained in the absence of AS or with larger aortic diameters ( $4.5-5$ and $\geq 5 \mathrm{~cm}$ subgroups).

\section{Validation of Elastic Fiber Thickness With Biomechanical Testing of Tissue Stiffness}

Biaxial mechanical testing of resected aortic wall specimens was used to interrogate the relationship between histopathology and mechanical behavior. A strong trend between circumferential tissue stiffness and a decrease in elastic fiber thickness was observed ( $r=-0.41 ; P=.06$; Figure 5).

\section{DISCUSSION}

Elastic fiber fragmentation in the aortic media is a hallmark of bicuspid aortopathy, leading to compromised aortic biomechanics, and ultimately, catastrophic dissection and rupture. ${ }^{1}$ Understanding the mechanisms underlying maladaptive outward aortic remodeling is crucial to risk stratification and individualized surgical management strategies. Recent studies implicate aberrant valve-mediated hemodynamics in the progression of BAV aortopathy, ${ }^{9,10,18,19}$ but its exact role in mediating histopathology and biomechanical changes remains unclear.

In this novel study we directly compared the magnitude of in vivo valve-mediated WSS in the proximal thoracic aorta with quantitative histopathology of patient with BAV aortopathy. In a large data set of 93 tissue samples from 27 patients, we observed that: (1) the magnitude of regional WSS and elastic fiber disruption is heterogeneous within the aorta of an individual patient with BAV; (2) aortic WSS correlates with regional elastic fiber thinning; (3) the association between WSS and regional histopathology is strongest with predominant AS and in mildly dilated aortas $(<4.5 \mathrm{~cm})$; and (4) elastic fiber thinning correlates with impaired tissue biomechanics.

Outward vascular remodeling can occur in response to increased WSS as an adaptive mechanism to decrease the effective WSS sensed by local endothelial cells and return regional WSS to a physiologic range.$^{20}$ Importantly, we previously documented that BAV patients do not show expected decreases in regional WSS after aortic dilatation. ${ }^{20}$ Similarly, we found in the present study that WSS was significantly increased in patients with BAV compared with healthy volunteers with TAV at all circumferential locations but the LC. Compared with TAV, abnormal BAV cusp architecture might preclude normalization of valvemediated WSS despite progressive outward aortic remodeling. Consequently, BAV aortopathy might manifest as a more severe and progressive aortopathy than patients with TAV with similar proximal aortic WSS. The present study supports, but does not confirm, a causative role for aortic WSS in the progression of BAV aortopathy.

Valve-mediated WSS might be a particularly important trigger for pathogenesis in early stages (when the aorta is smaller) and in the presence of predominant AS. A causative role for underlying genetic connective tissue defects cannot be dismissed. Although genetic etiology continues to lack strong supporting evidence, our data might suggest that valve-mediated WSS is a less important trigger for exacerbating local histopathology in patients with predominant AR. These patients are suspected to have a genetic connective tissue defect and are more commonly younger with primarily root dilatation (root phenotype). ${ }^{21,22}$ Importantly, our findings of an association between magnitude of WSS and severity of local histopathology are most pronounced when stratified according to valve function and aortic diameter. The correlation between increased aortic WSS and decreased elastic fiber thickness is greater in patients with BAV-AS than when pooling all patients with BAV, and was not observed in patients with BAV-AR. These findings parallel previous studies in which BAV-mediated aortic hemodynamics are influenced by the type (AS or AR) and severity of valve dysfunction. ${ }^{18,23}$ Beyond hemodynamic differences, the behavior of these 2 BAV populations after isolated aortic valve replacement is dramatically different; the 10-fold higher risk of events in patients with BAV-AR leads to its association with the malignant root phenotype of BAV aortopathy. ${ }^{21,22}$ These findings are corroborated by demonstrably worse histopathology scoring of resected BAV-AR aortic tissue compared with BAV-AS. ${ }^{24,25}$ The latter is more stable over time, and within this BAV population the present study documents a significant inverse correlation between magnitude of WSS and histopathology. Girdauskas and colleagues $^{26}$ similarly reported an association between the degree of histopathology in resected BAV-AS tissue impinged upon by the systolic jet at the aortotomy site, but not in BAV-AR samples. Together, these findings suggest that BAV-AS confers a predictable pattern of WSSmediated regional aortopathy, and supports BAV-AS and BAV-AR aortas as discrete clinical entities with distinct mechanisms of aortopathy pathogenesis. Noninvasive WSS identification of diseased aorta might be preferable in selected BAV populations. Other noninvasive hemodynamic indices such as oscillatory shear index might best correlate in BAV-AR, and remain an important interest for exploration in future studies.

Herein, we report the strongest inverse correlation between aortic WSS and elastic fiber thickness in mildly dilated BAV aortas $(<4.5 \mathrm{~cm})$. Larger aneurysms might reflect more advanced disease with loss of sensitivity to obtain significant correlations in these patients. We 
TABLE 2. Medial elastic fiber thickness and WSS averaged in healthy volunteers and patients with BAV

\begin{tabular}{|c|c|c|c|}
\hline & Measurements, $n$ & $\begin{array}{c}\text { Median elastic fiber } \\
\text { thickness (interquartile } \\
\text { range), } \mu \mathrm{m}\end{array}$ & $\begin{array}{c}\text { Median WSS (interquartile } \\
\text { range), Pa }\end{array}$ \\
\hline \multicolumn{4}{|l|}{ Healthy volunteers } \\
\hline Anterior wall & 40 & - & $0.55(0.42-0.73)$ \\
\hline Posterior wall & 40 & - & $0.64(0.55-0.72)$ \\
\hline Greater curvature & 40 & - & $0.60(0.44-0.67)^{*}$ \\
\hline Lesser curvature & 40 & - & $0.63(0.51-0.84)$ \\
\hline \multicolumn{4}{|l|}{ All BAV patients } \\
\hline Anterior wall & 25 & $3.08(2.72-3.29)$ & $0.78(0.62-0.96)$ \\
\hline Posterior wall & 23 & $2.95(2.67-3.13)$ & $1.35(0.88-1.66) *, \ddagger$ \\
\hline Greater curvature & 30 & $2.71(2.56-2.88)^{*}, \ddagger$ & $1.20(0.99-1.38)^{*}, \ddagger$ \\
\hline Lesser curvature & 15 & $3.10(2.93-3.25)$ & $0.77(0.62-1.02)$ \\
\hline \multicolumn{4}{|c|}{ BAV subgroups according to AS } \\
\hline AS (AR or no-AR) & 73 & $2.94(2.67-3.13)$ & $1.10(0.76-1.46)$ \\
\hline No AS (predominant AR) & 20 & $2.76(2.54-3.16)$ & $0.93(0.78-1.14)$ \\
\hline \multicolumn{4}{|c|}{ BAV subgroups according to maximal aorta diameter, $\mathrm{cm}$} \\
\hline$<4.5$ & 33 & $2.94(2.69-3.20)$ & $0.99(0.77-1.37)$ \\
\hline $4.5-5$ & 42 & $2.95(2.66-3.12)$ & $1.01(0.77-1.33)$ \\
\hline$\geq 5$ & 18 & $2.70(2.56-3.02)$ & $1.14(0.78-1.45)$ \\
\hline
\end{tabular}

Data are stratified according to circumferential locations, AS and aorta diameter. For each, n denotes the total number of measurements. Bold numbers indicate $P<.05$ versus healthy volunteers. WSS, Wall shear stress; $B A V$, bicuspid aortic valve; $A S$, aortic valve stenosis, $A R$, aortic valve regurgitation. $* P<.05$ versus LC regions, within healthy volunteers and patients groups. $\ddagger P<.05$ versus anterior wall.

speculate that the observation of a significant correlation between the severity of histopathology and WSS in smaller-diameter aorta suggests a causative role for increased aortic WSS yielding increased elastic fiber degeneration in the early stages of disease. These data also

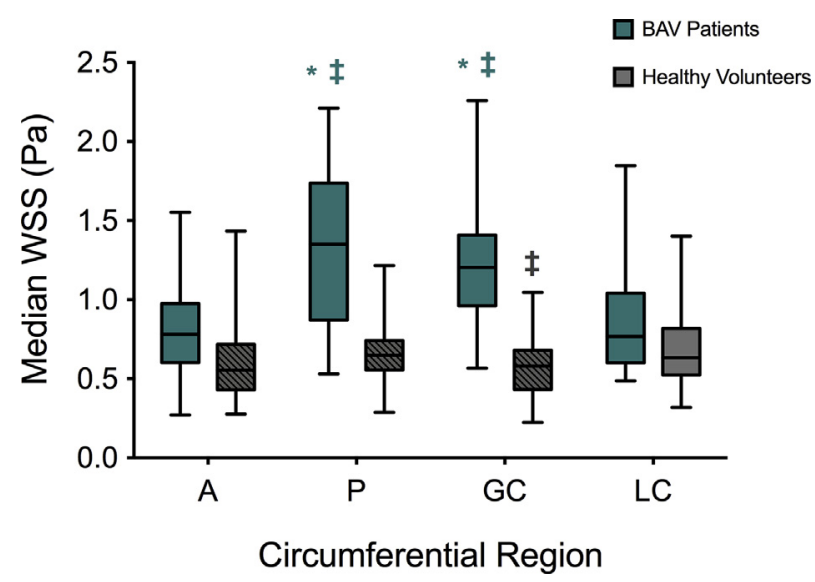

FIGURE 3. Aortic wall shear stress (WSS) assessed using 3-dimensional time-resolved phase-contrast magnetic resonance imaging with 3dimensional velocity encoding in patients with bicuspid aortic valve $(B A V ;$ blue $)$ and healthy volunteers ( gray) according to the circumferential location, while pooling the proximal and distal ascending aorta (A, Anterior; $P$, posterior, $G C$, greater curvature; and $L C$, lesser curvature). Columns illustrate median values whereas error bars indicate interquartile ranges. Hashed regions: $P<.05$ versus BAV patients. $* P<.05$ for comparison versus A; łagainst $\mathrm{LC}$, within each group. highlight the increasing appreciation that aortic size might not always predict the risk of aortic events or aortopathy severity. ${ }^{27}$ Our data show that subgroups of BAV patients with aorta $<4.5 \mathrm{~cm}$ (below current resection thresholds) might have substantially increased regional WSS with more severe histopathology that can be identified using 4D flow MRI. Further studies are needed to determine if such patients should be screened preoperatively and have more aggressive prophylactic resection at the time of aortic valve replacement. The correlation of WSS with simple histopathology for larger aortae with more advanced stages of disease might require more robust tools for discrimination such as biomechanical energy loss. ${ }^{28}$ Our future work is aimed at such study design. We document that tissue from the GC and P is subjected to increased aortic WSS as compared with the LC and A in patients with BAV. Similar to others, we show elastic fiber thinning is greatest at the $\mathrm{GC},{ }^{29}$ yet in the context of the correlations reported herein and before, ${ }^{10}$ the GC is not the only region to exhibit substantial aortopathy expression. These data support the emerging concept of regional heterogeneity within the BAV aorta: hemodynamics, ${ }^{9,18,19}$ smooth muscle cell origin and function, ${ }^{30}$ protease expression, ${ }^{10,31}$ oxidative stress, $^{32}$ and histopathology. ${ }^{10,26,29}$ Raaz and colleagues ${ }^{14}$ showed in a murine model that regional variations in vascular remodeling might propagate aortopathy, and the present findings of regional WSS-mediated histopathological differences within the same aorta might similarly contribute to the progression of BAV aortopathy. 


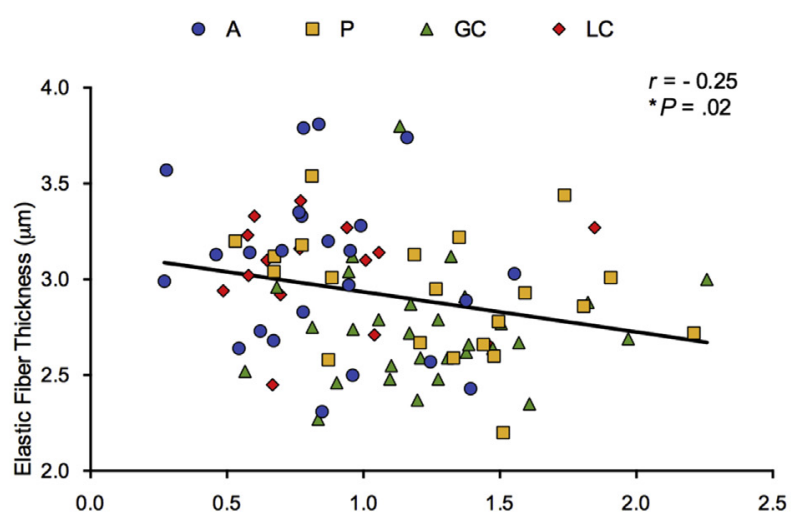

A Median WSS (Pa)
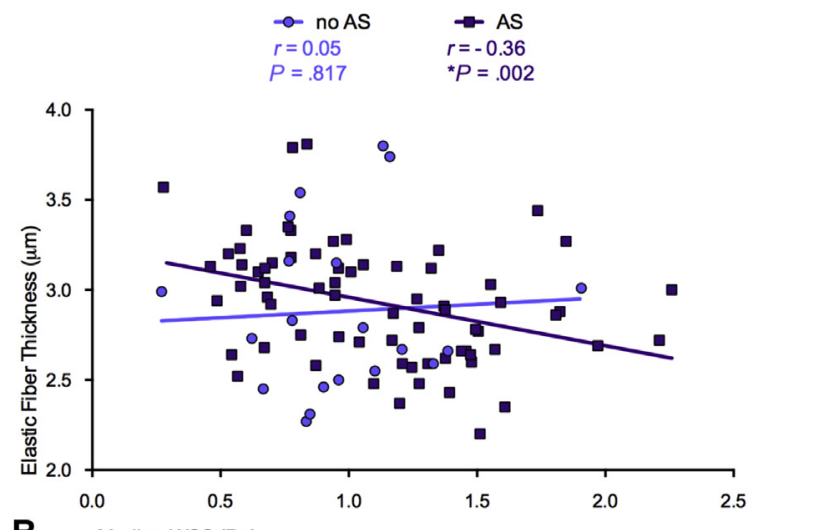

B $\quad$ Median WSS $(\mathrm{Pa})$

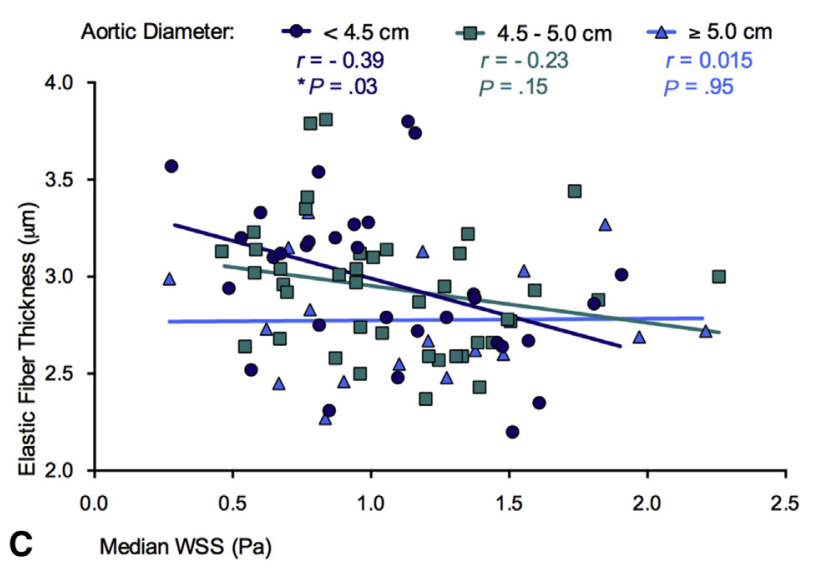

FIGURE 4. Correlations for comparison between aortic elastic fiber thickness and wall shear stress (WSS): (A) over all patients and all locations ( $A$, Anterior; $P$, posterior, $G C$, greater curvature; and $L C$, lesser curvature); (B) in patients with aortic valve stenosis (AS, squares); (C) in longitudinal ascending aortic locations with a diameter $<4.5 \mathrm{~cm}$ (circles). Correlation coefficients $r, P$ values and lines of best fit are provided.

Aortic dissection or rupture is prevented by healthy biomechanical properties of aortic tissue. Forsell and colleagues $^{33}$ previously documented increased collagenrelated stiffness in aneurysmal BAV versus TAV aortic tissue. To validate elastic fiber thinning as a predictive

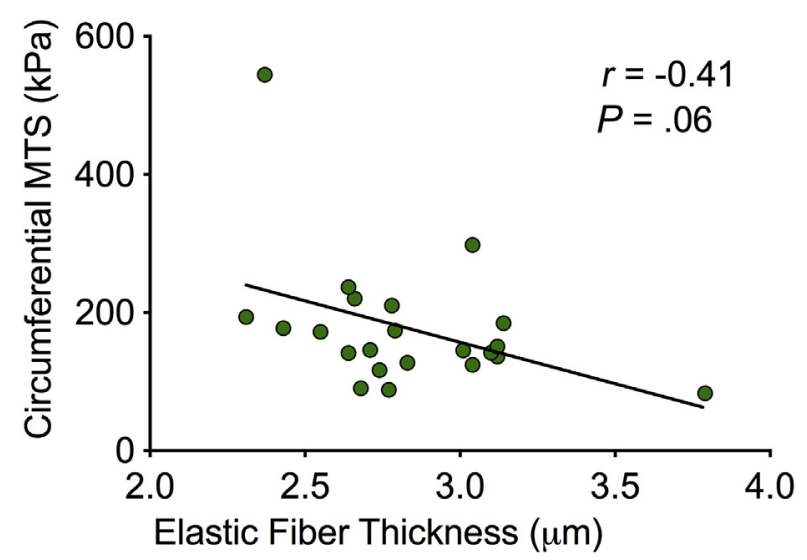

FIGURE 5. Correlations for comparison between aortic elastic fiber thickness and circumferential maximum tangential stiffness (MTS). Correlation coefficient $r, P$ values and line of best fit are provided.

biomarker of aortic disease, we investigated its relationship with the stiffness of the obtained tissue samples. Regional aortic tissue stiffness precedes aortic growth in animal models of human disease and is validated as a critical biomechanical marker of aortopathy progression. ${ }^{14}$ We report for the first time that decreased elastic fiber thickness corresponds with increased circumferential tissue stiffness in human BAV aorta. These data support elastic fiber thinning as a histopathologic biomarker of BAV aortopathy that can be influenced by local aortic valve-mediated WSS.

Our novel findings provide 2 important contributions to the BAV literature. First, the correlation of in vivo aortic WSS with quantitative tissue histopathology severity provides unique insight into BAV aortopathy, and supports a critical role for hemodynamics in its pathogenesis. However, we and others believe $e^{6,34}$ that hemodynamics likely acts synergistically with an inherent extracellular matrix dysregulation and other cellular mechanisms. Second, we provide data to support an association between nonvalidated hemodynamic imaging biomarkers with validated tissue metrics of aortopathy (elastic fiber architecture and biomechanical stiffness). Long-term clinical outcomes study will be required in a larger patient cohort to validate noninvasive hemodynamic imaging biomarkers, such as WSS, as a promising tool for risk stratification and individualized resection strategies for patients with BAV. ${ }^{35}$

We cannot exclude the effects of selection bias on our analysis and conclusions. Tissue resection performed at the operating surgeon's discretion and resection of smaller aortae might reflect unmeasured effects, such as intraoperative observations of tissue quality or thickness. Although previously examined in patients with $\mathrm{BAV},{ }^{10,12}$ elastic fiber thickness represents the first quantitative aortopathy metric correlated to aortic WSS and biomechanical stiffness in BAV aortopathy. However, it is possible that 
our methods are not sufficiently sensitive to discern subtle differences in the diffuse aortopathy of moderately- and severely-dilated aortas. Development of other quantitative indices might be needed with advanced microscopy. ${ }^{29}$ In addition, we report a moderate correlation between WSS and elastic fiber thickness, which could be attributable to spatial registration between histology and imaging, with spatial scales and resolutions that differ. An association between histopathology and tissue stiffness from a small sample using biaxial mechanical testing was shown. Future studies with more comprehensive constitutive modeling of tissue biomechanics and more advanced multiple linear regression models are warranted to fully explore the relationships between in vivo WSS and aortic histopathology investigated in this study. Finally, longitudinal clinical study of a larger cohort, along with stratification of patients according to the severity of AS and valve morphology ( $\mathrm{Si}$ evers classification), is needed to validate aortic WSS as a predictor of disease progression and clinically relevant acute aortic events for discrete BAV populations.

\section{CONCLUSIONS}

Increased aortic valve-mediated WSS is associated with elastic fiber thinning in patients with BAV, particularly in the presence of AS and for earlier stages of aortopathy. Elastic fiber thinning correlates with impaired tissue biomechanics. These novel findings implicate valve-mediated hemodynamics in the progression of BAV aortopathy and encourage further validation of noninvasive hemodynamic imaging biomarkers for risk stratification and development of individualized aortic resection strategies beyond diameter alone.

\section{Conflict of Interest Statement}

Authors have nothing to disclose with regard to commercial support.

\section{References}

1. Fedak PW, Verma S, David TE, Leask RL, Weisel RD, Butany J. Clinical and pathophysiological implications of a bicuspid aortic valve. Circulation. 2002; 106:900-4.

2. Fedak PW. Bicuspid aortic valve and the specialty clinic: are your patients at risk? Cardiol Young. 2017;27:411-2.

3. Verma S, Yanagawa B, Kalra S, Ruel M, Peterson MD, Yamashita MH, et al. Knowledge, attitudes, and practice patterns in surgical management of bicuspid aortopathy: a survey of 100 cardiac surgeons. J Thorac Cardiovasc Surg. 2013; 146:1033-40.e4.

4. Hardikar AA, Marwick TH. The natural history of guidelines: the case of aortopathy related to bicuspid aortic valves. Int J Cardiol. 2015;199:150-3.

5. Opotowsky AR, Perlstein T, Landzberg MJ, Colan SD, O'Gara PT, Body SC, et al. A shifting approach to management of the thoracic aorta in bicuspid aortic valve. J Thorac Cardiovasc Surg. 2013;146:339-46.

6. Girdauskas E, Borger MA, Secknus MA, Girdauskas G, Kuntze T. Is aortopathy in bicuspid aortic valve disease a congenital defect or a result of abnormal hemodynamics? A critical reappraisal of a one-sided argument. Eur J Cardiothorac Surg. 2011;39:809-14.

7. Markl M, Schnell S, Wu C, Bollache E, Jarvis K, Barker AJ, et al. Advanced flow MRI: emerging techniques and applications. Clin Radiol. 2016;71:779-95.
8. Tanweer O, Wilson TA, Metaxa E, Riina HA, Meng H. A comparative review of the hemodynamics and pathogenesis of cerebral and abdominal aortic aneurysms: lessons to learn from each other. J Cerebrovasc Endovasc Neurosurg. 2014; 16:335-49.

9. Barker AJ, Markl M, Bürk J, Lorenz R, Bock J, Bauer S, et al. Bicuspid aortic valve is associated with altered wall shear stress in the ascending aorta. Circ Cardiovasc Imaging. 2012;5:457-66.

10. Guzzardi DG, Barker AJ, Van Ooij P, Malaisrie SC, Puthumana JJ, Belke DD, et al. Valve-related hemodynamics mediate human bicuspid aortopathy: insights from wall shear stress mapping. J Am Coll Cardiol. 2015;66:892-900.

11. Nishimura RA, Otto CM, Bonow RO, Ruiz CE, Skubas NJ, Iii JP, et al. 2014 AHA/ACC guideline for the management of patients with valvular heart disease: a report of the American College of Cardiology/American Heart Association task force on practice guidelines. Circ. 2014;129:2440-92.

12. Bauer M, Pasic M, Meyer R, Goetze N, Bauer U, Siniawski H, et al. Morphometric analysis of aortic media in patients with bicuspid and tricuspid aortic valve. Ann Thorac Surg. 2002;74:58-62.

13. Rosin NL, Agabalyan N, Olsen K, Martufi G, Gabriel V, Biernaskie J, et al. Collagen structural alterations contribute to stiffening of tissue after splitthickness skin grafting. Wound Repair Regen. 2016;24:263-74.

14. Raaz U, Zöllner AM, Schellinger IN, Toh R, Nakagami F, Brandt M, et al Segmental aortic stiffening contributes to experimental abdominal aortic aneurysm development. Circulation. 2015;131:1783-95.

15. Sievers HH, Schmidtke C. A classification system for the bicuspid aortic valve from 304 surgical specimens. J Thorac Cardiovasc Surg. 2007;133:1226-33.

16. Kari FA, Fazel SS, Mitchell RS, Fischbein MP, Miller DC. Bicuspid aortic valve configuration and aortopathy pattern might represent different pathophysiologic substrates. J Thorac Cardiovasc Surg. 2012;144:516-7.

17. Potters WV, van Ooij P, Marquering H, VanBavel E, Nederveen AJ. Volumetric arterial wall shear stress calculation based on cine phase contrast MRI. J Magn Reson Imaging. 2015;41:505-16.

18. Bissell MM, Hess AT, Biasiolli L, Glaze SJ, Loudon M, Pitcher A, et al. Aortic dilation in bicuspid aortic valve disease: flow pattern is a major contributor and differs with valve fusion type. Circ Cardiovasc Imaging. 2013;6:499-507.

19. Mahadevia R, Barker AJ, Schnell S, Entezari P, Kansal P, Fedak PW, et al. Bicuspid aortic cusp fusion morphology alters aortic three-dimensional outflow patterns, wall shear stress, and expression of aortopathy. Circulation. 2014;129:673-82.

20. van Ooij P, Markl M, Collins JD, Carr JC, Rigsby C, Bonow RO, et al. Aortic valve stenosis alters expression of regional aortic wall shear stress: new insights from a 4-dimensional flow magnetic resonance imaging study of 571 subjects. $J$ Am Heart Assoc. 2017;6:e005959.

21. Girdauskas E, Rouman M, Disha K, Espinoza A, Misfeld M, Borger MA, et al. Aortic dissection after previous aortic valve replacement for bicuspid aortic valve disease. J Am Coll Cardiol. 2015;66:1409-11.

22. Della Corte A, Bancone C, Quarto C, Dialetto G, Covino FE, Scardone M, et al Predictors of ascending aortic dilatation with bicuspid aortic valve: a wide spectrum of disease expression. Eur J Cardiothorac Surg. 2007;31:397-405.

23. Shan Y, Li J, Wang Y, Wu B, Barker AJ, Markl M, et al. Aortic shear stress in patients with bicuspid aortic valve with stenosis and insufficiency. J Thorac Cardiovasc Surg. 2017;153:1263-72.e1.

24. Roberts WC, Vowels TJ, Ko JM, Filardo G, Hebeler RF, Henry AC, et al. Comparison of the structure of the aortic valve and ascending aorta in adults having aortic valve replacement for aortic stenosis versus for pure aortic regurgitation and resection of the ascending aorta for aneurysm. Circulation. 2011;123:896-903.

25. Girdauskas E, Rouman M, Borger MA, Kuntze T. Comparison of aortic media changes in patients with bicuspid aortic valve stenosis versus bicuspid valve insufficiency and proximal aortic aneurysm. Interact Cardiovasc Thorac Surg. 2013;17:931-6.

26. Girdauskas E, Rouman M, Disha K, Fey B, Dubslaff G, von Kodolitsch Y, et al Morphologic and functional markers of aortopathy in patients with bicuspid aortic valve insufficiency versus stenosis. Ann Thorac Surg. 2017;103:49-57.

27. Coady MA, Rizzo JA, Hammond GL, Mandapati D, Darr U, Kopf GS, et al. What is the appropriate size criterion for resection of thoracic aortic aneurysms? J Thorac Cardiovasc Surg. 1997;113:476-91.

28. Chung J, Lachapelle K, Wener E, Cartier R, De Varennes B, Fraser R, et al. Energy loss, a novel biomechanical parameter, correlates with aortic aneurysm size and histopathologic findings. J Thorac Cardiovasc Surg. 2014;148:1082-8; discussion: 1088-9.

29. Tsamis A, Phillippi JA, Koch RG, Chan PG, Krawiec JT, D’Amore A, et al. Extracellular matrix fiber microarchitecture is region-specific in bicuspid aortic valveassociated ascending aortopathy. J Thorac Cardiovasc Surg. 2016;151:1718-28.e5. 
30. Jiao J, Xiong W, Wang L, Yang J, Qiu P, Hirai H, et al. Differentiation defect in neural crest-derived smooth muscle cells in patients with aortopathy associated with bicuspid aortic valves. EBioMedicine. 2016;10: 282-90.

31. Ikonomidis JS, Ruddy JM, Benton SM, Arroyo J, Brinsa TA, Stroud RE, et al. Aortic dilatation with bicuspid aortic valves: cusp fusion correlates to matrix metalloproteinases and inhibitors. Ann Thorac Surg. 2012;93:457-63.

32. Kotlarczyk MP, Billaud M, Green BR, Hill JC, Shiva S, Kelley EE, et al. Regional disruptions in endothelial nitric oxide pathway associated with bicuspid aortic valve. Ann Thorac Surg. 2016;102:1274-81.

33. Forsell C, Björck HM, Eriksson P, Franco-Cereceda A, Gasser TC. Biomechanical properties of the thoracic aneurysmal wall: differences between bicuspid aortic valve and tricuspid aortic valve patients. Ann Thorac Surg. 2014;98:65-71.

34. Michelena HI, Prakash SK, Della Corte A, Bissell MM, Anavekar N, Mathieu P, et al. Bicuspid aortic valve identifying knowledge gaps and rising to the challenge from the international bicuspid aortic valve consortium (BAVCON). Circulation. 2014;129:2691-704.

35. Fedak PW, Verma S. Bicuspid aortopathy and the development of individualized resection strategies. J Thorac Cardiovasc Surg. 2014;148:2080-1.

Key Words: bicuspid aortic valve, aortopathy, wall shear stress, 4D flow MRI

Readers who found these articles interesting may also like to read the following papers found in recent and future issues of our sister publications, Seminars in Thoracic and Cardiovascular Surgery and Operative Techniques in Thoracic and Cardiovascular Surgery!

\section{Adult: Aortic Valve}

ORIGINAL SUBMISSION: Single Dose Del Nido Cardioplegia in Minimally Invasive Aortic Valve Surgery. Daniel Ziazadeh. Semin Thoracic Surg 2017: 471-476

Editorial Commentary: Is "As Good" Good Enough? James I. Fann. Semin Thoracic Surg 2017: 477-478

ORIGINAL SUBMISSION: Aortic Root Reconstruction and Valve Repair During Acute Type A Aortic Dissection Repair. Jared P. Beller. Oper Tech Thorac Cardiovasc 2017: 80-90

ORIGINAL SUBMISSION: Repair of the Bicuspid Aortic Valve. Ulrich Schneider. Oper Tech Thorac Cardiovasc 2017: 91-109 
APPENDIX E1. TESTING DETAILS, ANALYSIS, AND MODELING OF THE EXPERIMENTAL DATA Tensile Mechanical Testing, Analysis, and Modeling

Planar biaxial mechanical testing was conducted as before for skin tissue. ${ }^{\mathrm{E} 1}$ Aortic specimens were thawed at $4^{\circ} \mathrm{C}$ overnight before testing. Samples were trimmed to approximately $1 \mathrm{~cm}^{2}$ and mounted in a 4-motor biaxial testing apparatus (ElectroForce Systems, TA Instruments, Springfield, Mo) using 4 hooks attached to each side with 6.0 braided silk suture. Five dots were made on the luminal surface of each specimen using a surgical marker for camera tracking. Samples were hydrated for the duration of testing with $37^{\circ} \mathrm{C} 1 \mathrm{X}$ phosphate-buffered saline. Two $22.5 \mathrm{~N}$ load cells were preloaded to $0.01 \mathrm{~N}$ to remove residual slack in the sutures before testing. Motor control captured global specimen movements during testing.

Specimens were conditioned for 10 cycles per protocol at a rate of $0.3 \mathrm{~mm} / \mathrm{s}$ at $40 \%$ global strain; data were collected at sampling frequency of $200 \mathrm{~Hz}$ on the 10th cycle. Five protocols with differing tension ratios per axis were run: $1: 1-$ equibiaxial loading of each axis to $40 \%$ strain; $1: 0.75-1$ axis pulled to $40 \%$ strain and the other to only $75 \%$ of that; $1: 0.5 ; 0.5: 1$; and $0.75: 1$. Modeling and analysis of data were conducted as before, ${ }^{\text {E1 }}$ and maximum tangential stiffness (in kilopascals), the maximum slope of the stressstrain curve, was computed along the circumferential direction, the same direction as histopathology analysis.

\section{MRI Acquisitions}

MRI exams were conducted using a MAGNETOM 1.5T Aera, Avanto or Espree $(\mathrm{n}=32)$ and 3T Skyra $(\mathrm{n}=15)$ scanners (Siemens Medical Systems, Erlangen, Germany). 4D flow MRI data were acquired in a sagittal volume encompassing the thoracic aorta. Respiration gating was performed using a 16-mm acceptance window size navigator placed on the lung-liver interface. Generalized autocalibrating partial parallel acquisition imaging (GRAPPA) along the phase encoding direction (y) was used with a reduction factor of $\mathrm{R}=2$ ( 24 reference lines) to accelerate the acquisition. Other sequence parameters were as follows: repetition time $=4.8 \pm 0.1 \mathrm{~ms}$; echo time $=2.4 \pm 0.1 \mathrm{~ms}$; acquisition matrix $=160$ to $192 \times 80$ to 116 ; isotropic pixel in-plane spacing $=2.2 \pm 0.2$ (range, 1.7-2.9) $\mathrm{mm}$; slice thickness $=2.7 \pm 0.3$ (range, 2.2-3.5) $\mathrm{mm} ; 2$ k-space segments per cardiac time frame; temporal resolution $=38.8 \pm 1.2$ (range, 36.0-41.6) ms; receiver band width $=445$ to $460 \mathrm{~Hz}$ per pixel. A flip angle of $7^{\circ}$ was used for healthy volunteers, whereas it was set to $15^{\circ}$ in patients with $\mathrm{BAV}$, who received injection of a gadolinium-based contrast agent $(0.03 \mathrm{~mol} / \mathrm{kg}$ Ablavar; Lantheus Medical Imaging, N Billerica, Mass; or $0.1-0.2 \mathrm{mmol} / \mathrm{kg}$ Gadavist or Magnevist; Bayer, Leverkusen, Germany; or 0.1-0.2 $\mathrm{mmol} / \mathrm{kg}$ Multihance; Bracco Diagnostics Inc, Monroe Township, NJ). Finally, an encoding sensitivity Venc $=150 \mathrm{~cm} / \mathrm{s}$ was used in controls, and Venc varied from 150 to $400 \mathrm{~cm} / \mathrm{s}$ in patients with BAV depending on the presence and severity of AS.

Assessment of Left Ventricular Function and Aortic Dimensions. Left ventricular end-systolic and end-diastolic (EDV) volumes were measured with conventional contouring of ECG-gated cine balanced steady state free precession short-axis images, while including the papillary muscles and chamber trabecula, using QMass v7.2 (Medis, Leiden, The Netherlands). Stroke volume $=$ EDV minus endsystolic volume; and ejection fraction $=$ stroke volume divided by EDV were calculated. Aortic diameter measurements were obtained from contrast-enhanced magnetic resonance angiography images using the open-source, free Digital Imaging and Communications in Medicine medical image viewer Horos (2015, http://www.horosproject.org/; on the basis of OsiriX). Sinuses of Valsalva diameter were the maximal value among the 3 sinus-to-sinus measurements, including the external walls. Diameter at all other locations was the maximal value over 2 orthogonal measurements in a reformatted plane orthonormal to the aorta, including the external walls. ${ }^{\mathrm{E} 2}$

\section{D Flow MRI Data Preprocessing}

For each 4D flow MRI data set, preprocessing was first applied using a previously described Matlab program (MathWorks), ${ }^{\mathrm{E} 3}$ including eddy current correction, background noise suppression, and velocity aliasing unwrapping. A 3-D phase-contrast magnetic resonance angiogram (PC-MRA) was computed by multiplying absolute velocity by magnitude images and averaging over all cardiac phases, ${ }^{\mathrm{E}}$ to segment the aortic volume (Mimics; Materialize, Leuven, Belgium) and subsequently mask the flow velocities.

\section{References}

E1. Rosin NL, Agabalyan N, Olsen K, Martufi G, Gabriel V, Biernaskie J, et al. Collagen structural alterations contribute to stiffening of tissue after split-thickness skin grafting. Wound Repair Regen. 2016;24:263-74.

E2. Trinh B, Dubin I, Rahman O, Ferreira Botelho MP, Naro N, Carr JC, et al. Aortic volumetry at contrast-enhanced magnetic resonance angiography. Invest Radiol. 2017:52:216-22.

E3. Schnell S, Entezari P, Mahadewia RJ, Malaisrie SC, McCarthy PM, Collins JD, et al. Improved semiautomated 4D flow MRI analysis in the aorta in patients with congenital aortic valve anomalies versus tricuspid aortic valves. J Comput Assist Tomogr. 2016;40:102-8. 
TABLE E1. Location of resected aortic tissue samples in the 27 patients with BAV, according to longitudinal zone and circumferential quadrant

\begin{tabular}{lcc}
\hline \multirow{2}{*}{$\begin{array}{c}\text { Circumferential } \\
\text { quadrant, } \mathbf{n}\end{array}$} & \multicolumn{2}{c}{ Longitudinal zone } \\
\cline { 2 - 3 } & $\begin{array}{c}\text { Proximal } \\
\text { (26 patients) }\end{array}$ & $\begin{array}{c}\text { Distal AA } \\
\text { (5 patients) }\end{array}$ \\
\hline Anterior & 23 & 2 \\
Posterior & 21 & 2 \\
Greater curvature & 25 & 5 \\
Lesser curvature & 12 & 3 \\
\hline $\begin{array}{l}\text { n denotes the total number of samples available over all patients. AA, Ascending } \\
\text { aorta. }\end{array}$
\end{tabular}

